\title{
TITLE:
}

\section{FORMATION AND DECAY OF RUDIMENTARY PENUMBRA AROUND A PORE}

\section{$\operatorname{AUTHOR}(S)$ :}

Watanabe, Hiroko; Kitai, Reizaburo; Otsuji, Kenichi

\section{CITATION:}

Watanabe, Hiroko ...[et al]. FORMATION AND DECAY OF RUDIMENTARY PENUMBRA AROUND A PORE. The Astrophysical Journal 2014, 796(2):

77.

\section{ISSUE DATE:}

2014-12-01

URL:

http://hdl.handle.net/2433/192763

RIGHT:

(C)2014. The American Astronomical Society. 


\title{
FORMATION AND DECAY OF RUDIMENTARY PENUMBRA AROUND A PORE
}

\author{
Hiroko Watanabe ${ }^{1}$, Reizaburo Kitai ${ }^{2,3}$, and Kenichi Otsuji ${ }^{4}$ \\ ${ }^{1}$ Unit of Synergetic Studies for Space, Kyoto University, Yamashina-ku, Kyoto 607-8417, Japan; watanabe@kwasan.kyoto-u.ac.jp \\ ${ }^{2}$ Kwasan and Hida Observatories, Kyoto University, Yamashina-ku, Kyoto 607-8417, Japan \\ ${ }^{3}$ Bukkyo University, Kitaku, Kyoto 603-8301, Japan \\ ${ }^{4}$ Solar Observatory, National Astronomical Observatory, Mitaka, Tokyo 181-8588, Japan \\ Received 2014 May 17; accepted 2014 September 17; published 2014 November 10
}

\begin{abstract}
We analyze the evolution of a pore in the active region NOAA 10940 using the data obtained by the Hinode satellite on 2007 February 3 . The pore we analyzed showed the formation of a rudimentary penumbra structure, succeeded by an abrupt disappearance after about $5 \mathrm{hr}$. The pore had an approximate radius of $3.5 \mathrm{Mm}$ and a total magnetic flux of $3.0 \times 10^{19} \mathrm{Mx}$, which is a little smaller than the necessary magnetic flux for penumbral formation supposed by Rucklidge et al. $\left(1-1.5 \times 10^{20} \mathrm{Mx}\right)$. Our observation describes a rare phenomenon which was in the unstable phase between a pore and a sunspot. The area of the dark umbra gradually decreased when the rudimentary penumbral filaments formed the penumbral structure, meaning that the penumbra develops at the expense of the umbral magnetic flux. This statement was confirmed by a rough estimation of the magnetic flux variation observed by the Hinode Fe I magnetogram. Five hours after the formation phase, the decay phase began. In this decaying phase, multiple opposite polarity patches are found to appear in the exterior of the pore (a different location from the penumbra formation site). We interpret these opposite polarities as signatures of the horizontal magnetic field, which preferably appears in the course of the unstable reconfiguration of the magnetic field structure. During the course of the disappearance of the penumbra, the horizontal penumbral field seems to become vertical because of the dark umbral area that recovered by about $10 \%$.
\end{abstract}

Key words: magnetic fields - Sun: photosphere - sunspots

Online-only material: color figures

\section{INTRODUCTION}

Solar pores are small sunspots without penumbra that are mostly observed within active regions. The structures of sunspots and pores are determined by the interaction between magnetic fields and convection. As the magnetic flux of the pore increases, the magnetic field at its outer edge becomes more inclined to the vertical (Simon \& Weiss 1970) and eventually becomes unstable to filamentary instability (Hurlburt \& Rucklidge 2000; Tildesley \& Weiss 2004). The resulting instability that interacted with the turbulent motion of surrounding granular convection leads to the production of a highly inclined field, i.e., penumbra, in a typical timescale of a few hours. However, it is difficult to predict when the penumbral formation occurs, as it takes place when the pore solution loses stability at a bifurcation point (Rucklidge et al. 1995) in the magnetic flux versus the radius plane. A sinking force to the inclined magnetic field is necessary to keep the penumbral structure from rising against the magnetic buoyancy, which is known as downward flux pumping (Thomas et al. 2002; Weiss et al. 2004; Brummell et al. 2008). On the other hand, the decay mechanism of the pore is considered to be the "turbulent erosion" process (Petrovay \& Moreno-Insertis 1997). The magnetic flux gradually leaks out and disperses into the adjacent downflowing network intergranular lanes (Cameron et al. 2007).

The velocity field around the pore has an intimate relation with the surrounding granules. Sobotka et al. (1999) found that the granular motions in the vicinity of pores show mesogranular flows. The annular zone of about $2^{\prime \prime}$ around the pore is characterized by the horizontal radially inward flow, and, as manifested by these motions, granular fragments sometimes penetrate into the pore. Another phenomenon observed around the pore is the so-called "finger-like structure" reported in Bellot Rubio et al. (2008). Finger-like structures are known to have a $1-2 \mathrm{~km} \mathrm{~s}^{-1}$ upflow and a horizontal magnetic field, and they are preferentially observed at the decaying phase of a spot. The simulation by Rempel et al. (2009) showed an average outflow of about $1-2 \mathrm{~km} \mathrm{~s}^{-1}$ in the penumbral region and weak inflows at the inner edges of penumbral filaments. The simulation by Rempel (2011) showed fast outflows exceeding $8 \mathrm{~km} \mathrm{~s}^{-1}$ along the almost horizontal magnetic field.

Recently, the top boundary condition above the pore was highlighted in the reconfiguration of the penumbral structure. The simulation by Rempel (2012) showed that the penumbra could disappear, or form, within about 30 minutes after the top boundary condition is changed. This is supported by the observation of penumbral disappearance during the flares (Wang et al. 2012), or the observation of the chromospheric precursor of the penumbral formation (Shimizu et al. 2012).

In this paper, we analyze a pore in the active region NOAA 10940 observed by the Hinode satellite (Tsuneta et al. 2008; Suematsu et al. 2008). Thanks to Hinode, we obtained stable, high-resolution images of the pore that are free from atmospheric seeing disturbances. Interestingly, the pore showed the formation of a rudimentary penumbra, succeeded by the abrupt decay of the structure within $10 \mathrm{hr}$ of the observation. We use the filtergrams of the $G$-band, $\mathrm{Ca}$ II $\mathrm{H}$, and Fe I $6302 \AA$ A line magnetograms of the Solar Optical Telescope (SOT). In the following sections, we describe the details of the observation and the data calibration in Section 2, analyze the evolution of the pore in Section 3, and finally summarize our results, and provide some interpretation, in Section 4. 

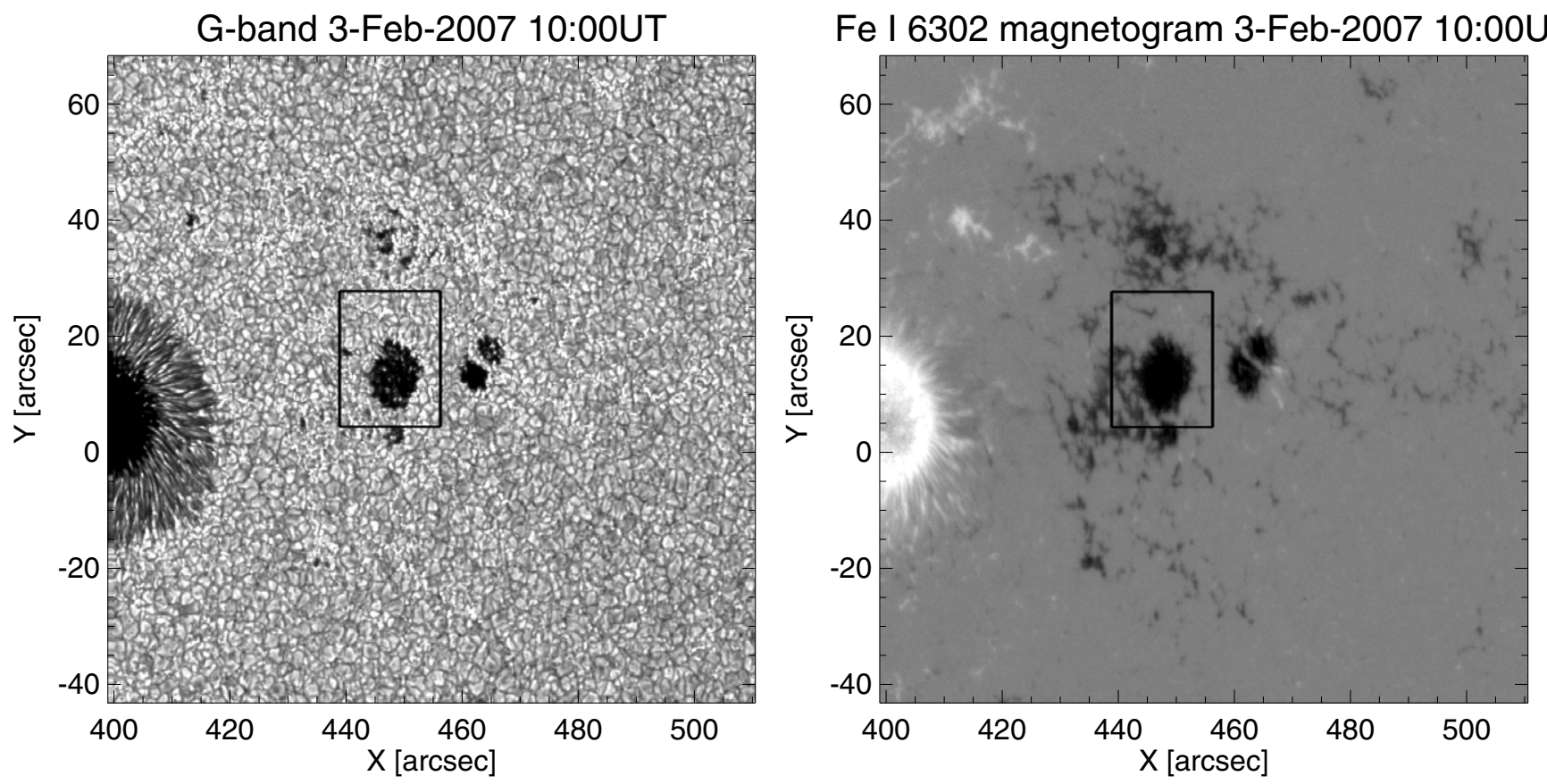

Figure 1. Hinode G-band and magnetogram images of NOAA 10940. The main sunspot to the left (east) has positive polarity, while the pores to the right (west) have negative polarity. The black rectangle shows the cropped FOV of Figure 3.

\section{OBSERVATION AND DATA CALIBRATION}

Hinode tracked the active region NOAA 10940 from 10:43 UT on 2007 February 2 until 06:01 UT on 2007 February 6. The active region NOAA 10940 was a dipole-type region with preceding pores and a succeeding main sunspot. We are interested in one of the preceding pores that shows the formation and decay of the penumbral structure successively on February 3 (Figure 1). For our purpose, we focus on the time period between 10:00 UT and 20:55 UT on February 3. The pore was located at (S5.8, W28.4) at 10:00 UT, and (S5.1, W34.9) at 20:55 UT. During this period, the SOT obtained sequences of filtergrams of the $G$-band, Ca II $\mathrm{H}$, and line-of-sight (LOS) magnetograms of the Fe I $6302.5 \AA$ line (created from Stokes $V / I$ with an offset of $-120 \mathrm{~m} \AA$ in the shuttered mode). The temporal cadence was mostly 1.5 minutes, but sometimes switched into a lower cadence (7 minutes) observation. The spatial pixel size was 0 '! 11 for the $G$-band and Ca II $\mathrm{H}$, and 0.16 for the LOS magnetogram. For our analyses, the $\mathrm{Fe}$ I magnetograms were spatially rescaled to have the same pixel size as the other two.

The total number of the sequence of filtergrams is 322 . After the dark field subtraction and the flat fielding, $G$-band images were carefully coaligned by finding the displacement that gave the maximum cross-correlation between consecutive frames around the pore. Then, the same displacement was applied to the Ca II $\mathrm{H}$ data set. Next, we manually found the spatial offset between the $G$-band and Fe I magnetograms using the first images of the sequences, and applied the same displacement found for the $G$-band temporal sequence to the $\mathrm{Fe}$ I sequence. This way, we get all of the three filtergrams coaligned with each other within an accuracy of less than 1 pixel.

To estimate the magnetic flux variation of the pore, we calculate the conversion factor $\beta$ between the observed narrowband filter imager (NFI) Stokes $V / I$ value and the LOS field strength $\left(B_{\|}\right)$adopting a linear relation $B_{\|}=\beta V / I$ (Chae et al. 2007). In the derivation of $\beta$, we used the SOT Spectropolarimeter (SP) data as references. The SP data were pro-

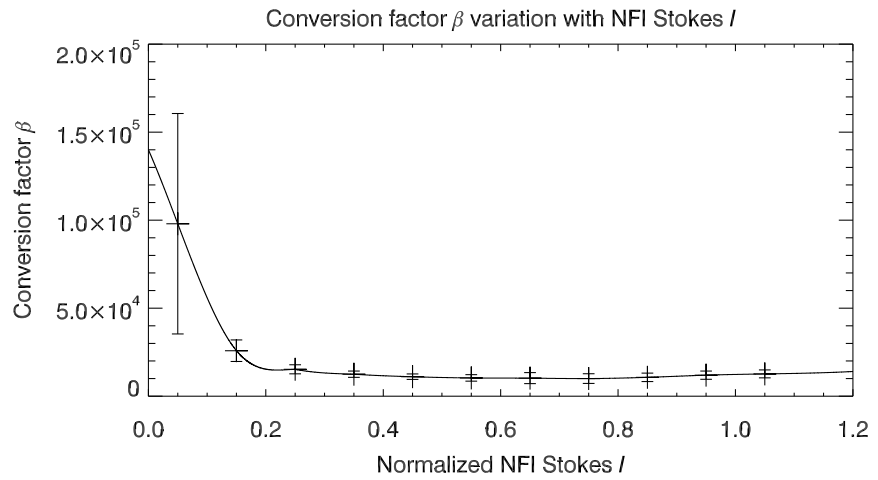

Figure 2. Plot of the conversion factor $\beta$ as a function of the normalized NFI Stokes $I\left(I_{\text {norm }}\right)$. The solid line shows the mean value of $\beta$ derived from the NFI and SP data sets between 2006 November and 2007 March. The error bars were calculated from the standard deviation for all the data sets within a certain NFI Stokes $I$ bin.

cessed to three-dimensional magnetic field strength using the full Milne-Eddington inversion. On the other hand, we constructed "scanned" NFI Stokes $I$ and $V$ images as follows. First, we picked up the narrow rectangular portions from the consecutive NFI data according to the SP slit position. The height of the clipped rectangles corresponds to the height of the SP field of view (FOV) and the different widths of the rectangles correspond to the slit positions which are observed at the closest timing out of consecutive NFI observations. Next, we put the clipped rectangles side by side to mimic the SP data. When the temporal cadence of NFI is high enough, the constructed "scanned" NFI images can be regarded as the images cospatial with the reference SP data. Using a number of NFI and SP data obtained between 2006 November and 2007 March, we derived the dependency of the conversion factor $\beta$ against the NFI Stokes $I\left(I_{\text {norm }}\right)$, normalized by quiet area intensities (Figure 2). In the plot, in the small Stokes $I$ area $\left(I_{\text {norm }}<0.1\right)$, the conversion factor $\beta$ has a large error $(\sim 60 \%)$. This error arises from the NFI Stokes $V / I$ saturation in the umbral area. For the 

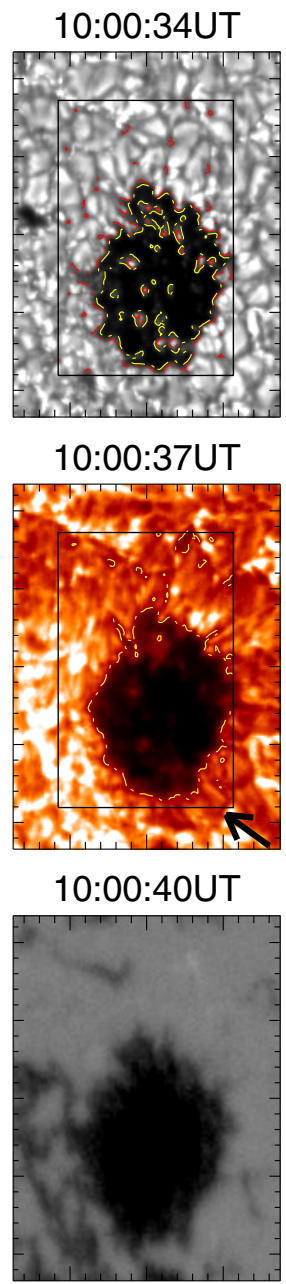
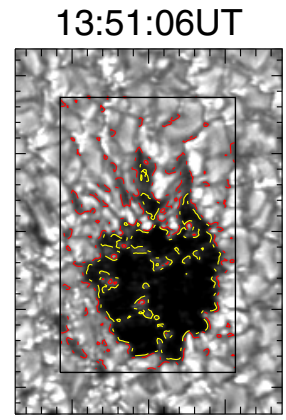

13:51:09UT
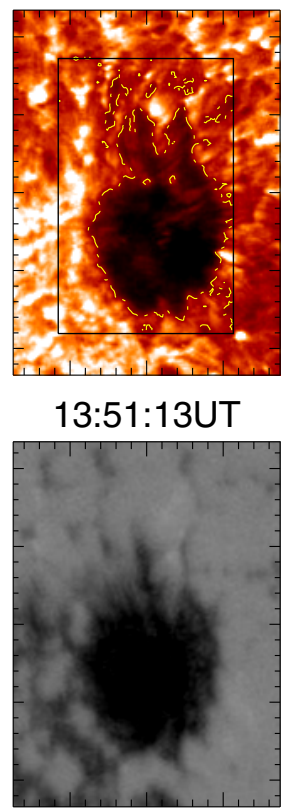

15:09:08UT

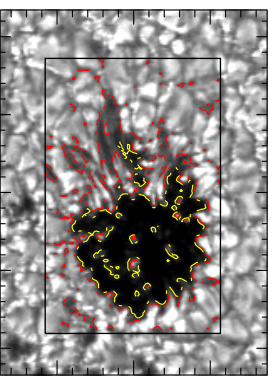

15:09:12UT
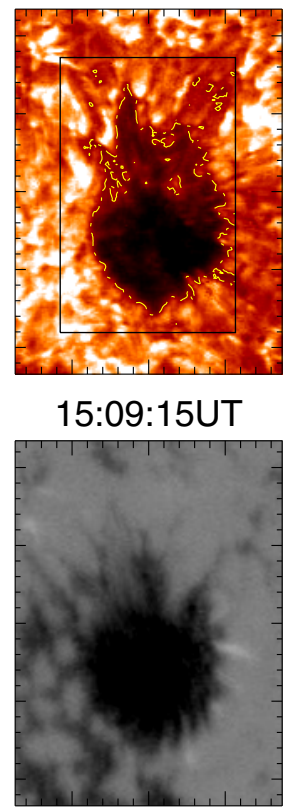

17:11:03UT

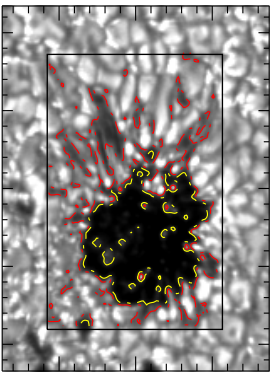

17:11:06UT

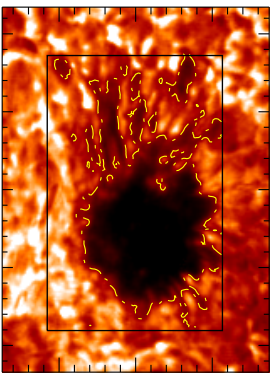

17:11:10UT

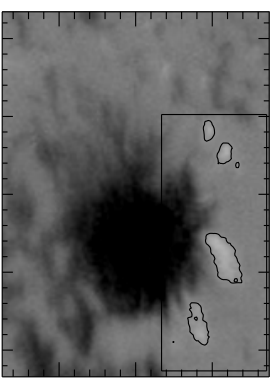

19:24:33UT

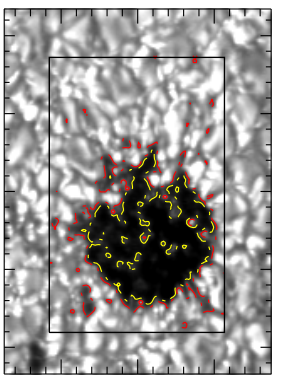

19:24:36UT

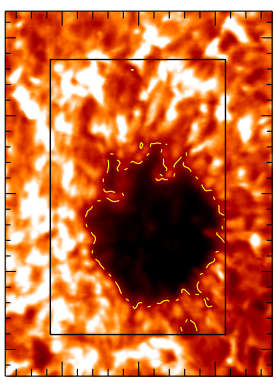

19:24:39UT

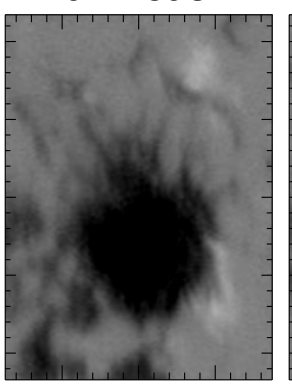

20:55:36UT

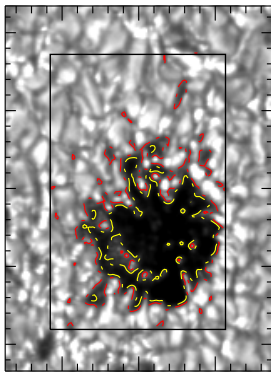

20:55:39UT

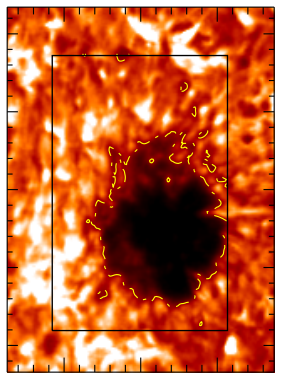

20:55:46UT

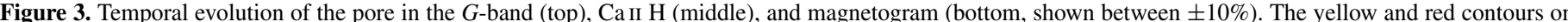

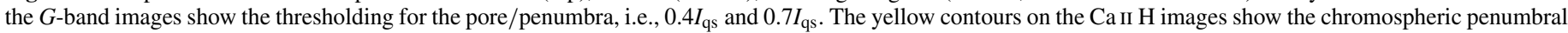

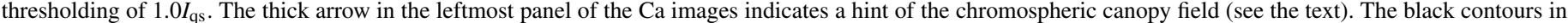
the middle bottom panel show the opposite polarity patches described in Section 3.3.

(A color version of this figure is available in the online journal.)

larger $I_{\text {norm }}$ area, $\beta$ is almost constant around $\sim 1 \times 10^{4}$, and the relative error decreases to $\sim 20 \%$. For the analysis of the total flux evaluation, we applied interpolated $\beta$ values to convert the observed NFI Stokes $V / I$ to $B_{\|}$. We neglected the umbral area with $\left(I_{\text {norm }}<0.2\right)$ in the derivation of $B_{\|}$because of the large error.

In order to create a better contrast of fine scale structures, we corrected for the instrumental stray light using the pointspread function (PSF) of the SOT Broadband Filter Imager (Wedemeyer-Böhm 2008). We used the ideal $G$-band PSF for the main mirror of SOT (FWHM of 0.'18), provided by Y. Suematsu (2012, private communication). The deconvolution was carried out iteratively by updating the current estimate of the image using an IDL maximum likelihood routine (max_likelihood.pro in the AstroLib package; Richardson 1972). The Ca II H images were also corrected similarly, because ideal PSFs can be scaled with wavelength. The convolved and deconvolved images were subsequently normalized to the quiet-Sun intensity.

\section{ANALYSIS}

Figure 3 shows the temporal evolution of the pore in the $G$-band, CaII H, and Fe I 6302 magnetogram. During our $11 \mathrm{hr}$ observation, the rudimentary penumbra to the north of the pore started to form, and, interestingly, it abruptly began to shrink and completely disappeared. The apparent speed of the elongation of a single penumbral filament measured by eye in the $G$-band was about $2-3 \mathrm{~km} \mathrm{~s}^{-1}$, while the overall penumbral structure extended its boundary with a moderate speed of about $0.5 \mathrm{~km} \mathrm{~s}^{-1}$. The length of the penumbral filament was $7^{\prime \prime}$ at maximum. In the chromosphere, brightenings are often observed at the outer edge of the penumbra. These values and characteristics are consistent with earlier studies (e.g., Shine et al. 1987). During the penumbral decay, the frequent appearance of opposite polarity patches was observed on the west side of the pore (black-contoured patches in the lowest row of Figure 3), and, simultaneously, many small-scale fragmented patterns appeared in the intensity images.

We studied the areal variation of the umbra/penumbra using $G$-band and CaII $\mathrm{H}$ images (Section 3.1), the variation of the photospheric total magnetic flux (Section 3.2), and the appearance of opposite polarity patches (Section 3.3) in order to discuss the mechanism of the penumbral formation and decay.

\subsection{Umbral/Penumbral Area Variation}

We adopted the intensity threshold method in order to know the pore area variation, including the development of 


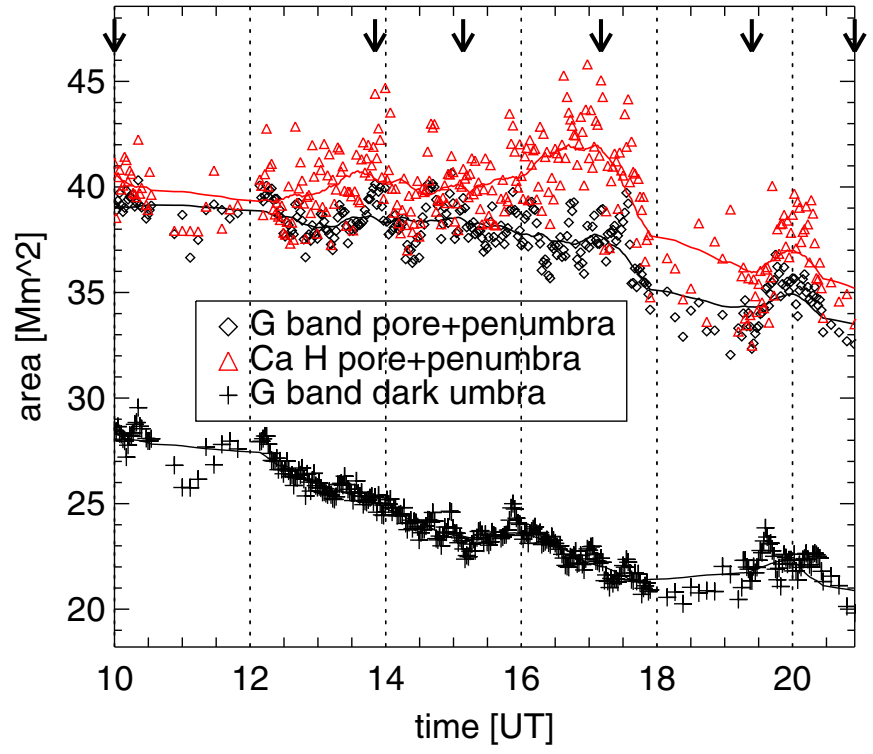

Figure 4. Temporal variation of the area of pore + penumbra in the $G$-band (black diamond), pore + penumbra in $\mathrm{Ca}$ II $\mathrm{H}$ (red triangle), and dark umbra in the $G$-band (black plus). The solid lines show smoothed variation with about a one hour width. The black arrows at the top of the plot indicate the timing of the frames in Figure 3.

(A color version of this figure is available in the online journal.)

the penumbral structure with time. The pore + penumbral area was defined by the area with intensity darker than $0.7 I_{\mathrm{qs}}$ for the $G$-band and $1.0 I_{\mathrm{qs}}$ for $\mathrm{Ca}$ II $\mathrm{H}\left(I_{\mathrm{qs}}\right.$ is the average quiet-Sun intensity). For reference, the dark umbral area was calculated to be the area darker than $0.4 I_{\mathrm{qs}}$ in the $G$-band. These thresholds were determined based on our by-eye inspection. By cropping the FOV small enough so that it contains the pore and the evolving penumbral structure, which is outlined by the black rectangle in Figure $3\left(11^{\prime \prime} .5 \times 17^{\prime \prime} .8\right)$, we minimized the contamination of other features. Figure 3 and its contours demonstrate the effectiveness of our intensity threshold method.

Figure 4 shows the plots of the area of the dark umbra and pore + penumbral area in both the $G$-band and Ca II H. At first, only the naked pore was seen both in the $G$-band and $\mathrm{Ca}$ II $\mathrm{H}$, and area of this initial naked pore was almost the same in both the $G$-band and $\mathrm{Ca}$ II H, i.e., $40 \mathrm{Mx}^{2}$. Please keep in mind that the Hinode's Ca II H images are contaminated by photospheric intensity from its broad filter transmission profile (Tsuneta et al. 2008). The area of the pore + penumbra was nearly constant until 17 UT, while the dark umbral area showed a clear decrease from $12 \mathrm{UT}$ to $18 \mathrm{UT}$. This means that the penumbral structure developed from $12 \mathrm{UT}$ at the expense of the umbral area. The area of dark umbra was about $28 \mathrm{Mx}^{2}$ at $12 \mathrm{UT}$ and decreased to about $21 \mathrm{Mx}^{2}$ at $18 \mathrm{UT}$. There was a small hump of umbral area around $20 \mathrm{UT}$ of about $10 \%$.

We notice the following three characteristics when comparing the pore + penumbral areas in the $G$-band and $\mathrm{Ca}$ II $\mathrm{H}$ along with an inspection of the movie. (1) The universal small surplus in the Ca II $\mathrm{H}$ area came from the exclusion of bright umbral dots (Louis et al. 2012) in the $G$-band images. (2) During the formation of the penumbral filament, the area surplus in Ca II $\mathrm{H}$ got larger, especially around $17 \mathrm{UT}$ when the penumbral structure was visually the largest. This area surplus was due to the widening of the filaments in $\mathrm{Ca}$ II $\mathrm{H}$, not the lengthening of the filaments. (3) We could not find any timing difference between the $G$-band and $\mathrm{Ca}$ II $\mathrm{H}$ both for the formation of the

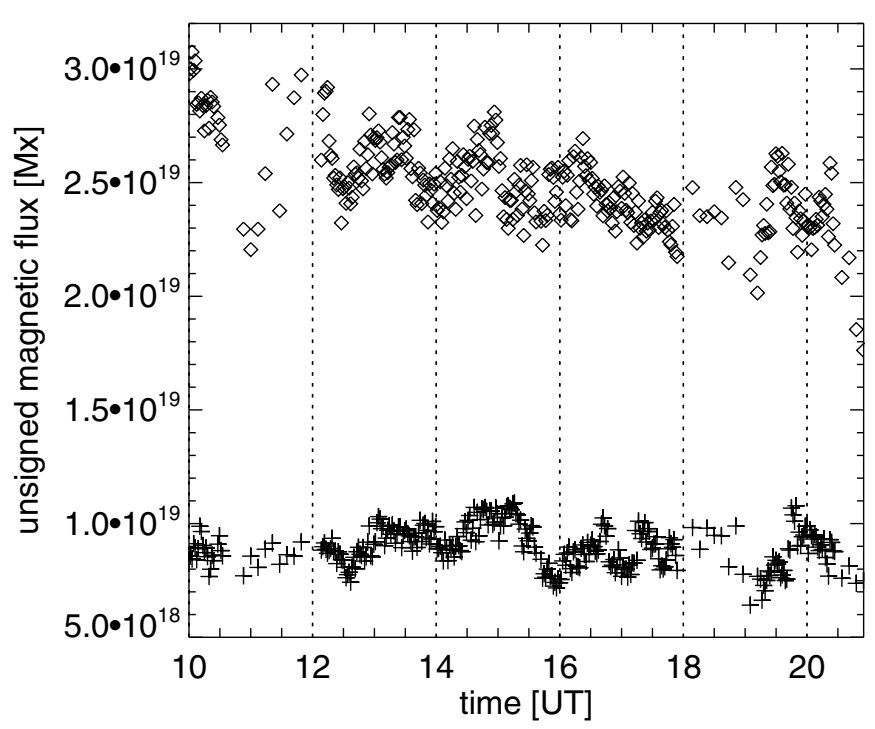

Figure 5. Temporal variation of the unsigned magnetic flux of the dark pore (diamond symbol) and the penumbra region (plus symbol). The definition of the area is based on the continuum (see the text).

penumbra (12 UT) and the onset of the abrupt decay of the penumbra (after $17 \mathrm{UT}$ ).

We also want to mention the possibility of the existence of a chromospheric canopy field, which was first reported by Shimizu et al. (2012). In the leftmost middle panel of Figure 3, an arrow points to the position of a partial dark "penumbra-like" structure. This looks similar to the dark annular zone in Shimizu et al. (2012), which they presumed to be the precursor of the photospheric penumbral structure. In our data, on the other hand, this dark "penumbra-like" zone was perceptible only during 10-13 UT, and no photospheric penumbra formation followed in this zone. We expect that this kind of dark annular zone is unstable, sometimes leading to the photospheric penumbral formation and sometimes not. The recent finding by Sobotka et al. (2013a, 2013b) of a super-penumbral structure above a naked pore may be related to this phenomena, though its temporal stability is not yet known.

\subsection{Magnetic Flux Variation}

The estimation of the total magnetic flux was performed using $B_{\|}$maps described in Section 2. The unsigned magnetic flux was calculated by the integration of unsigned $B_{\|}$over the area of interest. Please note that our calculation may underestimate the magnetic flux of the locally vertical umbral field because the LOS direction is not parallel to the local vertical direction. The area definition was based on the continuum intensity as is written in Section 3.1. The dark pore is the area darker than $0.4 I_{\mathrm{q} s}$, and penumbral area has an intensity between $0.4 I_{\mathrm{q} s}$ and $0.7 I_{\mathrm{q} s}$.

In Figure 5, we first find that the magnetic flux of this pore was about $3.0 \times 10^{19} \mathrm{Mx}$. This is a few factors smaller than the typical critical magnetic flux necessary for the penumbral formation (1-1.5 × 10 ${ }^{20} \mathrm{Mx}$ in Leka \& Skumanich 1998). Next, the total magnetic flux of the pore showed a nearly linear decrease during the penumbral formation (10-15 UT). The rate of the magnetic flux decrease was nearly the same as that of the areal decrease, which means that the average magnetic flux density in the pore did not change much during the penumbral formation. In fact, it was confirmed that the magnetic flux densities at the central part of the pore did not show significant 


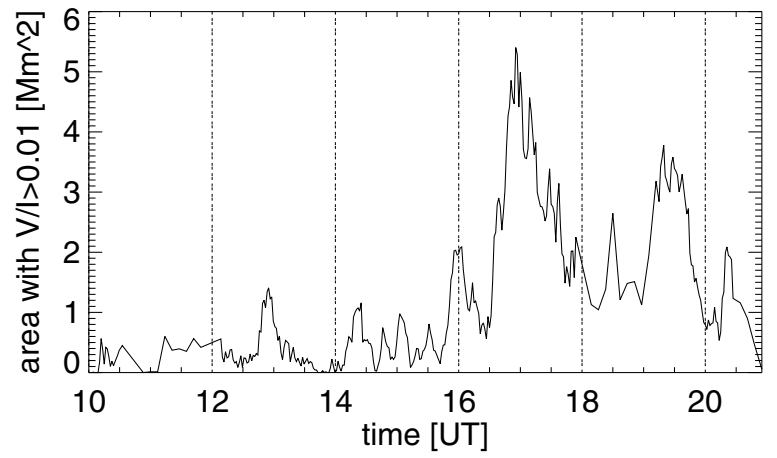

Figure 6. Temporal evolution of the area with $V / I>0.01$ within the region adjacent to the pore (see the text).

temporal variations. The increase of penumbral magnetic flux was nearly balanced with the decrease of the pore magnetic flux until around $15 \mathrm{UT}$.

\subsection{Opposite Polarity Patches}

We can see the diminishment of the area with the strong vertical magnetic field in the temporal series of the Fe I magnetogram shown in Figure 3, as the pore grew within the penumbral structure. The penumbral area showed weaker $V / I$ signals, demonstrating that the penumbral magnetic field was highly inclined. However, there was no magnetic flux concentrated at the outer edge of the penumbral formation area, which was reported in Shimizu et al. (2012). Even after the disappearance of the penumbral structure in the intensity images, such as in the last two panels in Figure 3, the weak $V / I$ signals to the north of the pore remains.

One remarkable thing is the emergence of opposite polarity patches (shown by the black contours in Figure 3 ) on the right side of the exterior of the pore. In Figure 6, we plot the area with $V / I>0.01$ within the rectangular region around the west (right) side of the pore, which is outlined in the fourth panel of the magnetograms in Figure 3.

In the first half of the period, we observed the transient appearance of opposite polarity patches around the outer edge of the pore. Typically, a single opposite polarity patch did not persist for more than 30 minutes. They appeared adjacent to the negative polarity pore in a rather elongated shape along the radial direction. The size of the patch was about $1 \mathrm{Mm}$.

The situation changed after 16 UT. Before 16 UT, there were one to three small patches existing simultaneously. Between $16 \mathrm{UT}$ and $18 \mathrm{UT}$, both the number and the size of opposite polarity patches increased sharply. Around the peak at 17 UT, more than five opposite polarity patches were seen. After their active emergence ceased around $18 \mathrm{UT}$, a large and loopshaped opposite polarity region appeared in the lower right side outside the pore after 19UT (elaborated in the following paragraphs). Most of these opposite polarity patches were not moving magnetic features, because they did not migrate radially outward from the main pore. However, some of them, like the small lower one in panel (c) of Figure 7, exhibited migration characteristics similar to a moving magnetic feature. Please refer to a recent study of moving magnetic features around a pore in Criscuoli et al. (2012).

A closer comparison of the opposite polarities with $G$-band images gives us insights into their physical origin. Figure 7 shows four different timings to demonstrate four types of their occurrences. In panel (a), an opposite polarity patch was

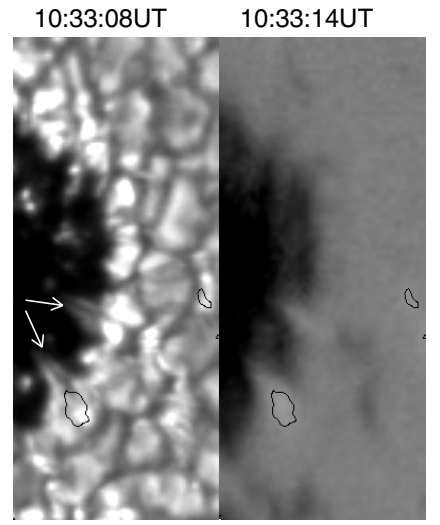

(a)

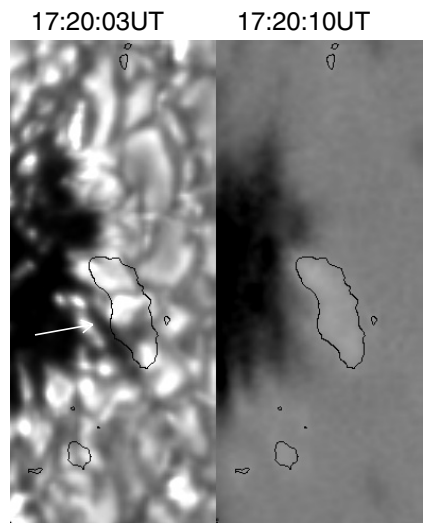

(c)

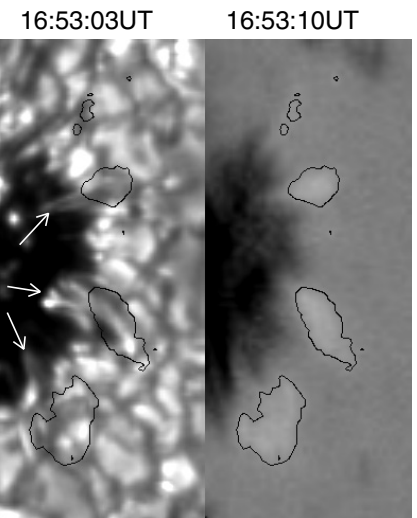

(b)

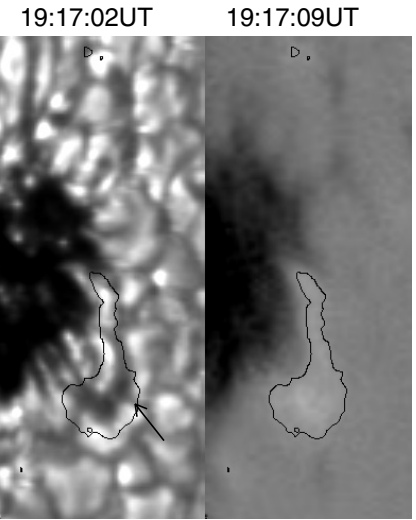

(d)
Figure 7.Zoomed in images of the western region of the pore in the $G$-band (left) and magnetogram (right). The black contours, commonly used in the $G$-band and magnetograms, outline the area with a magnetogram signal larger than 0.01 . The white and black arrows in the $G$-band images indicate the corresponding fragmented structures.

located at the outer edge of a bright granular fragment with a central dark lane, which was intruding on the dark part of the pore. This case is the most commonly observed throughout the observing period. The central dark lanes were not always observed, probably because of the insufficient spatial resolution. The intruding granular fragments decayed by shrinking and, at the same time, opposite polarities disappeared. These granular fragments are different from the penumbral grains in the sense that their tips did not continue to become isolated umbral dots (Watanabe et al. 2012).

Panels (b)-(d) depict somewhat special cases. The timing of case (b) corresponds to the sharp rise of the opposite polarity flux shown around 17 UT. During this period (approximately from 16:32 UT to 17:05 UT), larger sized ( $2^{\prime \prime}-3^{\prime \prime}$ diameter) opposite polarity patches were observed, together with the formation of a "transitory penumbra." The length of the filamentary structure was $3^{\prime \prime}-5^{\prime \prime}$. Panel (c) is chosen during the period when the amount of opposite polarity decreased sharply. This case is characterized by the appearance of a dark elongated structure in the $G$-band image and a broad region of opposite polarity (about $\left.2^{\prime \prime} \times 5^{\prime \prime}\right)$ located adjacent to the dark elongated structure. The dark elongated structure kept its identity for about $1 \mathrm{hr}$ between $17 \mathrm{UT}$ and $18 \mathrm{UT}$. The granules inside the opposite polarity regions showed turbulent motion and irregular shapes (abnormal granulation), but no systematic motion. In panel (d), there was a dark loop structure spanning part of the pore boundary. The dark loop structure was cospatial with opposite polarity. The loop structure (about $3^{\prime \prime} \times 3^{\prime \prime}$ ) kept its shape between 19:12 UT 
and 19:20 UT, and then it mingled with the intergranular lanes. The granules confined by the loop had an elongated shape but were not similar to penumbral filaments.

The opposite polarity patches that appear in white in our magnetogram may not represent a real positive polarity, i.e., dipped down to the surface, because the pore was located far from the disk center $\approx(\mathrm{S} 5, \mathrm{~W} 30)$. West (to the right in the image) is limbward, meaning the horizontal field was observed as white (positive) in our magnetogram.

\section{SUMMARY AND DISCUSSION}

We analyzed a rare phenomenon of transient penumbral filament formation around a pore. The lifetime of this phenomenon was about six hours or so. Leka \& Skumanich (1998) called the pores with short-lived or rudimentary penumbrae protospots. Thanks to the continuous and high-cadence data by the Hinode satellite, we observed the detailed evolution of the protospot and were successful in obtaining a plausible physical model of the phenomenon.

The main results are as follows.

1. The total magnetic flux of the pore was about $3.0 \times 10^{19} \mathrm{Mx}$, which is a few factors smaller than the previously reported critical magnetic flux necessary for the penumbral formation $\left(1-1.5 \times 10^{20} \mathrm{Mx}\right)$.

2. The area of dark umbra gradually decreased when the penumbral filaments were starting to extend. The area of dark umbra was about $40 \mathrm{Mx}^{2}$ at $12 \mathrm{UT}$ and about $21 \mathrm{Mx}^{2}$ at $18 \mathrm{UT}$.

3. The area of dark umbra recovered a bit (about 10\%) after the disappearance of the penumbra.

4. The penumbral filaments were wider in the chromosphere than in the photosphere, but their lengths were the same in both layers.

5. There were no timing differences between the chromosphere and the photosphere during the course of the formation and the decay of the penumbra.

6. Opposite polarity patches appeared transiently at the side of the pore that was different from the penumbral formation site. At the inner edge of the opposite polarity patches, there existed dark-cored elongated granules that were intruding into the pore.

7. Active emergence of opposite polarity patches, together with the transitory penumbral structure in the intensity images, were observed when the evolving penumbral structure turned into the decay phase.

8. There were relatively persistent and large dark structures with opposite polarities in various shapes during the decay phase of the penumbral structure.

Rucklidge et al. (1995) studied the equilibrium morphology of magnetic concentration on the solar surface and also the temporal evolution of its morphology when the magnetic flux is gradually accumulated. They found that there are two branches of equilibrium in the plane of the magnetic flux versus the radius of the concentration. One is the pore branch and the other is the spot branch. When the magnetic flux is accumulated, an initially stable pore state changes to a sunspot state with penumbra. From the simplified model calculation, they derived a critical radius of $1.8-3.5 \mathrm{Mm}$ or a critical magnetic flux of $1-1.5 \times 10^{20} \mathrm{Mx}$. The pore radius in our analysis stayed in this range throughout its evolution. Although the magnetic flux we measured is smaller than the theoretically predicted one, we expect that the total magnetic flux will have a similar value to that predicted if we include the perpendicular magnetic component, because the target pore was a bit far from the disk center. Therefore, we think that the observed pore was in a marginal state of transition with a simple magnetic configuration similar to the theoretical model, and that the transition from the pore state to the sunspot state occurred initially, and then returned back to the pore state again under the condition of the lack of additional magnetic flux supply.

Although the behavior of the observed pore is consistent with the result of Rucklidge et al. (1995), there remains some questions to be discussed. The location of the transient penumbral formation around the pore was restricted to the northern part of the pore. As there was a plage in the southeast direction and the same polarity pore (about 15" distant to the west), these surrounding magnetic structures may impede the transition to the penumbral formation.

Next, the surrounding magnetic configuration may control the speed and the height dependence of the transition. Shimizu et al. (2012) shows the existence of chromospheric precursor about 10 hours before the formation of photospheric penumbra, suggesting the formation of a magnetic canopy structure before the penumbral formation. In our case, the transition occurred in four hours without any chromospheric precursors, i.e., an abrupt transition. We think that the difference comes from the magnetic environment. In Shimizu's case, the region had larger magnetic flux, and probably had quieter chromospheric conditions than the pore in our case. Also, the speed and the mode of magnetic flux accumulation is, we think, an important factor for the formation of penumbrae. In the case discussed in Rezaei et al. (2012), the pore continuously accumulates additional magnetic flux from an adjacent emerging flux region, leading to a more mature and stable penumbral structure. Recently, we found several cases of penumbral formation from Hinode data (Kitai et al. 2014).

Therefore, we may conclude that the penumbral formation in the solar magnetic concentrations is basically due to the Rucklidge's mechanism or due to the magnetic pumping mechanism (Weiss et al. 2004), modified by the surrounding magnetic environment and magnetic accumulation.

The appearance of opposite polarity patches and corresponding intensity structures (dark-cored elongated granules and the dark long or loop-shaped structures) may characterize the observed signatures during the unstable state. Sobotka et al. (1999) also observed $2.8 \mathrm{Mm}$ long dark filament simultaneously with the transitory penumbra formation. It is worth noting that the motion of granules is rather turbulent at the site of opposite polarity patches, because the granules play an important role in keeping the stability of the field configuration (compare to the downward flux pumping process in Thomas et al. 2002).

The mechanism for the pore's decay is considered to be the magnetic flux gradually leaking out and dispersing in the down-flowing network intergranular lanes (called erosion process; Petrovay \& Moreno-Insertis 1997). However, the fact that the dark umbral area recovered indicates that, during the disappearance of the penumbra, the horizontal field lines returns to being vertical and becomes part of the pore again. This paper elaborates on magnetohydrodynamic behavior, in one example, showing the evolution of a pore in a marginal state between a pore and a sunspot. In the future, we expect further observations of the formation of the penumbra using the advent of highresolution satellite observations, and the addition of more clues to this unstable phenomena.

We thank Dr. Suematsu for his help with the deconvolution using the point-spread function of the Hinode SOT. We also 
thank the members of the Kwasan and Hida Observatories and Unit of Synergetic Studies for Space for fruitful discussions. We are supported by the Grant-in-Aid for JSPS Fellows, and by the grant-in-aid from the Japanese MEXT (PI: R. Kitai, No. 26400235). This work has made extensive use of the Hinode Solar Optical Telescope. We are grateful to the developers and observers of Hinode. Hinode is a Japanese mission developed and launched by ISAS/JAXA, with NAOJ as a domestic partner and NASA and STFC (UK) as international partners. It is operated by these agencies in cooperation with ESA and NSC (Norway).

\section{REFERENCES}

Bellot Rubio, L. R., Tritschler, A., \& Martínez Pillet, V. 2008, ApJ, 676, 698 Brummell, N. H., Tobias, S. M., Thomas, J. H., \& Weiss, N. O. 2008, ApJ, 686, 1454

Cameron, R., Schüssler, M., Vögler, A., \& Zakharov, V. 2007, A\&A, 474,261

Chae, J., Moon, Y.-J., Park, Y.-D., et al. 2007, PASJ, 59, 619

Criscuoli, S., Del Moro, D., Giannattasio, F., et al. 2012, A\&A, 546, A26

Hurlburt, N. E., \& Rucklidge, A. M. 2000, MNRAS, 314, 793

Kitai, R., Watanabe, H., \& Otsuji, K. 2014, PASJ, in press (arXiv:1407.4573)

Leka, K. D., \& Skumanich, A. 1998, ApJ, 507, 454

Louis, R. E., Mathew, S. K., Bellot Rubio, L. R., et al. 2012, ApJ, 752, 109
Petrovay, K., \& Moreno-Insertis, F. 1997, ApJ, 485, 398

Rempel, M. 2011, ApJ, 729, 5

Rempel, M. 2012, ApJ, 750, 62

Rempel, M., Schüssler, M., \& Knölker, M. 2009, ApJ, 691, 640

Rezaei, R., Bello González, N., \& Schlichenmaier, R. 2012, A\&A, 537, A19

Richardson, W. H. 1972, JOSA, 62, 55

Rucklidge, A. M., Schmidt, H. U., \& Weiss, N. O. 1995, MNRAS, 273, 491

Shimizu, T., Ichimoto, K., \& Suematsu, Y. 2012, ApJL, 747, L18

Shine, R. A., Title, A. M., Tarbell, T. D., et al. 1987, in NASA Conference Publication, Vol. 2483, Theoretical Problems in High-Resolution Solar Physics II, ed. G. Athay \& D. S. Spicer (Washington, DC: NASA), 133

Simon, G. W., \& Weiss, N. O. 1970, SoPh, 13, 85

Sobotka, M., Švanda, M., Jurčák, J., Heinzel, P., \& Del Moro, D. 2013a, JPhCS, 440, 012049

Sobotka, M., Švanda, M., Jurčák, J., et al. 2013b, A\&A, 560, A84

Sobotka, M., Vázquez, M., Bonet, J. A., Hanslmeier, A., \& Hirzberger, J. 1999, ApJ, 511, 436

Suematsu, Y., Tsuneta, S., Ichimoto, K., et al. 2008, SoPh, 249, 197

Thomas, J. H., Weiss, N. O., Tobias, S. M., \& Brummell, N. H. 2002, Natur, 420, 390

Tildesley, M. J., \& Weiss, N. O. 2004, MNRAS, 350, 657

Tsuneta, S., Ichimoto, K., Katsukawa, Y., et al. 2008, SoPh, 249, 167

Wang, H., Deng, N., \& Liu, C. 2012, ApJ, 748, 76

Watanabe, H., Bellot Rubio, L. R., de la Cruz Rodríguez, J., \& Rouppe van der Voort, L. 2012, ApJ, 757, 49

Wedemeyer-Böhm, S. 2008, A\&A, 487, 399

Weiss, N. O., Thomas, J. H., Brummell, N. H., \& Tobias, S. M. 2004, ApJ, 600,1073 